\title{
MRI-Compatible Grasping Force Sensor with an Inclined Double Parallel Structure using Fiber Optics*
}

\author{
Jumpei Arata ${ }^{\dagger}$, Shogo Terakawa ${ }^{\dagger}$, James Sulzer $^{\ddagger}$, Roger GasserT ${ }^{\ddagger}$ and Hideo Fujimoto ${ }^{\dagger}$
}

\begin{abstract}
Investigations of human motor control using functional magnetic resonance imaging (fMRI) are increasingly receiving attention, with applications in fields such as motor learning and rehabilitation. In these neuroscience studies, force and position sensors are used to control haptic devices and safely interact with the human motion in an MR environment. However, conventional force sensors such as strain gauges are known to cause electromagnetic interference originating from electrical cables, transducers, and electronics. Light transmission through optical fibers is one alternative that avoids these problems. Since optical fibers do not produce electromagnetic noise, they can be used in an MR environment without electromagnetic interference. In this paper, we propose a novel design of an MRI-compatible grasping force sensor based on these principles. The sensor structure was designed to fit into an MRI scanner with its inclined double parallel mechanism, and was specifically adapted to precision grip tasks. This paper presents the sensor design and preliminary characterization in a non-MR environment.
\end{abstract}

\section{Introduction}

In recent years, we have seen an increasing number of developments in magnetic resonance imaging (MRI)-compatible robotics[1,3]. These dedicated MRI-compatible robotic devices allow neuroscientists to investigate the brain mechanisms of manipulation and motor learning (e.g., to improve rehabilitation therapies). However, the development of these MRI-compatible robotic devices requires careful consideration of safety and electromagnetic compatibility constraints.

First of all, an MR system generates a strong magnetic field with an associated spatial gradient, switching gradient fields and radio frequency pulses to obtain the image. MRI-compatible devices must not pose a safety hazard to the subject or patient in the MRI scanner or to the operator. Second, to avoid interference, which commonly results in image degradation, these robotic devices must be compatible with the magnetic field and must not emit electromagnetic noise. Finally, we must also consider the limited space in the MRI scanner. The space, accessibility and

\footnotetext{
* Manuscript Received Date: June 6, 2012

$\dagger$ Department of Computer Science and Engineering, Graduate School of Engineering, Nagoya Institute of Technology; Gokisocho, Showa-ku, Nagoya, Aichi 4668555, JAPAN

$\ddagger$ Rehabilitation Engineering Laboratory, Institute of Robotics and Intelligent Systems, ETH Zurich; LEO B

9.1, Leonhardstrasse 27, 8092 Zurich, SWITZERLAND Key Words: MRI compatibility, force sensor, sensor mechanism.
}

safety constraints produce the most significant challenges in the development of MRI-compatible robotic devices. Generally, ferromagnetic materials cannot be used in an MRI scanner because strong attraction forces occur due to the spatial gradient of the magnetic field. Electronic devices should be kept away from the MRI scanner to avoid capturing the electromagnetic noise generated from the scanner and to avoid interference with the MRI from the electronic noise generated by the electronic devices. Additionally, eddy currents induced in conducing materials can result in mechanical and thermal effects, which could potentially hurt the subject or patient.

To overcome these challenges, we propose a new grasping force sensor to be used for human motor control investigations with functional MRI (fMRI). Conventional force sensors such as strain gauges are known to cause electromagnetic interference with the equipment's electrical cables, transducers, and electronics, which may go unnoticed unless detailed compatibility tests are performed. Light transmission through optical fibers is one alternative that avoids these problems[1,10]; therefore, fiber optics are used in the developed sensor. In addition, to deal with the accessibility in the MRI scanner, we developed a compliant probe based on an inclined double parallel structure. This mechanism minimizes the dimensions of the sensor and positions the sensing axis perpendicular to the optical fiber, thereby avoiding a complex cable setup in the MRI scanner and avoiding damage to the optical fiber. This paper describes the developed MRI-compatible grasping sensor, its novel design, the design optimization analysis, and a pre- 
liminary characterization in a non-MR environment.

In this paper, related research on MRI-compatible force sensors is discussed in Section 2. In Section 3, the developed sensor design is presented. The design analysis is described in Section 4, and the preliminary characterization in a non-MR environment is discussed in Section 5. Finally, the conclusions are presented in Section 6.

\section{Related Research}

Recently, much research on MRI/fMRI-compatible force sensors has been presented[1]. Tan et al. developed a 3-axis force sensor for medical applications that use MRI/fMRI[4]. The presented device was made using plastic materials and an optical fiber sensor to make the sensor MRI-compatible. Puangmali et al. developed a 3-axis force sensor to realize force feedback for MRI-guided minimally invasive surgery [5]. The presented sensor has a flexible beam, which allows the system to measure 3 -axis force on the surgical tool's tip. Takahashi et al. developed an optical 6 -axis force sensor with elastic frames to be used in investigations of the brain mechanism[6]. Turkseven et al. presented a 1-DOF MRI-compatible force sensor that can be converted into a multi-DOF force sensor[7]. Tokuno et al. developed a 1-DOF force sensor with an optical lens[8]. This sensor is based on a parallel structure, which allows the lens to have 1-DOF displacement. Polygerinos et al. developed an optical force sensor that can be mounted on the tip of an active catheter[9]. Park et al. applied a Fiber Bragg Grating sensor to a grasping mechanism to measure the force[10]. Gassert et al. developed a 3-DOF force and a 1-DOF torque sensor using optical fibers to measure the reflected light intensity[1]. They also described the requirements for the development of MRIcompatible force/torque sensors.

Most of these sensors are based on elastic body deformation, which allows us to measure the displacement resulting from an applied force using fiber optics. The mechanism that uses these elastic bodies, commonly called a compliant mechanism, plays a significant role in overcoming the challenges described in Section 1. In conventional mechanisms, a revolute joint, which commonly consists of a revolute shaft and a bearing, is typically used. Revolute joints do have advantageous characteristics, such as high-speed rotation and a rigid structure. However, revolute joints typically require lubrication and high-precision machining to minimize mechanical backlash in highprecision robots. In a compliant mechanism, one or more elastic structures deform to transmit or transform the power source (instead of using conventional revolute joints) [11-13]. Also, there is no backlash, no need of lubrication, and no mechanical wear. In addition, a compliant mechanism can be easily integrated into a compact, lightweight, and simple structure compared to conventional mechanisms. On the other hand, parallel mechanisms[14] generally have

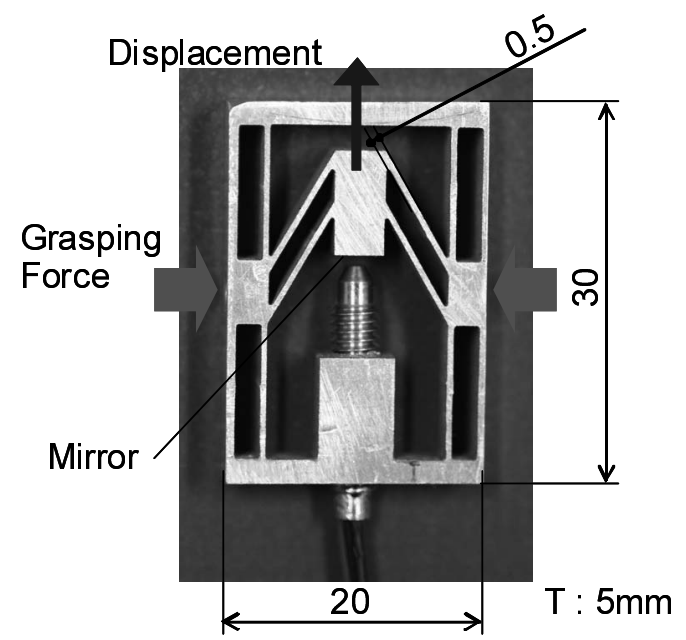

Fig. 1 Structure of the developed sensor

the advantages of high rigidity, output accuracy and backdrivability thanks to their multi-legged structure and fixed actuators. Since compliant mechanisms inherently have lower accuracy, the integration of a compliant parallel mechanism is a rational approach (e.g., a compliant parallel structure used as the force sensor[8]).

In this study, we introduce a compliant mechanism into an MRI-compatible sensor structure. Using the compliant mechanism in a parallel structure, the deformation can be constrained to a linear motion that can be measured using fiber optics. We propose inclined double parallel structure as a novel sensor structure, to develop a MRI-compatible grasping sensor, in taking into account the MRI environment. The novel structure can be effectively introduced to simplify the guiding of the optical fiber and placement of the device in the limited space or the MRI bore. Such specialization has not been made in previously presented studies. Therefore, the main contribution of the paper is the novel sensor structure.

\section{Developed Grasping Force Sen- sor}

In this section, the developed MR-compatible grasping force sensor is described. The sensor was developed to be used for human motor control investigations with fMRI. An overview of the developed sensor is given in Fig. 1. The developed sensor has one DOF of grasping, consists of a novel parallel structure, and a fiber optic sensor is used for the measurement.

\subsection{Fiber Optic Sensor}

As described in Section 1, careful consideration is needed to develop an MRI-compatible sensor. First, we chose a fiber optic sensor device based on the reflected light intensity measurement requirements (Baumer Electric, Switzerland, illustrated in Fig. 2). The specifications of the fiber optic sensor are described in Table 1. The fiber optic sensor emits light from the LED to the attached mirror on the sen- 


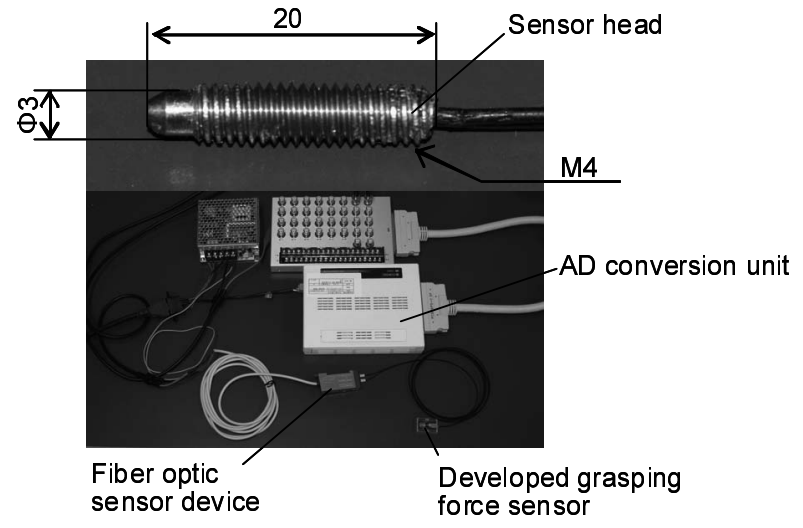

Fig. 2 Components of the fiber optic sensor

Table 1 Specifications of the fiber optic sensor

\begin{tabular}{|l|l|}
\hline Sensor head & FUE999C1004 \\
\hline Sensor base & FWDK 10U84Y0 \\
\hline Cable length & $10 \mathrm{~m}$ \\
\hline $\begin{array}{l}\text { Range of sensing } \\
\text { distance }\end{array}$ & $10 \mathrm{~mm}$ \\
\hline Light source & Pulsed red LED \\
\hline Wave length & $680 \mathrm{~nm}$ \\
\hline $\begin{array}{l}\text { Response time } \\
\text { release time }\end{array}$ & Adjustable from 1 to $50 \mathrm{~ms}$ \\
\hline Sensor head size & $\phi 4 \times 20(\mathrm{~L}) \mathrm{mm}$ \\
\hline Sensor base size & $10(\mathrm{~W}) \times 29.7(\mathrm{H}) \times 60(\mathrm{D}) \mathrm{mm}$ \\
\hline
\end{tabular}

sor structure through the optical fiber. Then, the reflected light is received by the sensor through the different channels of the optical fiber. When displacement occurs between the sensor head and the mirror, it can be measured by the change in light intensity. Since the sensor is made from a small piece of brass and only measures the light intensity, it is not affected by the MR environment. The electrical parts of the sensor, such as the amplifier, can be located at a sufficient distance away from the scanner (typically in the control room) so as to not affect to the MRI image. Therefore, the fiber optic sensor is MRI-compatible.

\subsection{Inclined Double Parallel Structure}

The developed inclined double parallel structure (Fig. 1) is composed of a pair of vertical parallelograms and a pair of inclined parallelograms, which are positioned symmetrically in the structure. The fiber optic sensor head is fixed to the bottom using an M4 screw, and a mirror is attached to the moving part located at the center of the structure and supported by the pair of inclined parallelograms.

The grasping force applied from the sides deforms the vertical parallelograms, resulting in a horizontal deformation of the pair of vertical parallelograms (relative to the sensor head). The horizontal deformation then propagates to the pair of inclined parallelograms, and the inclined parallel structure constrains the moving part to allow it to deform perpendicularly toward

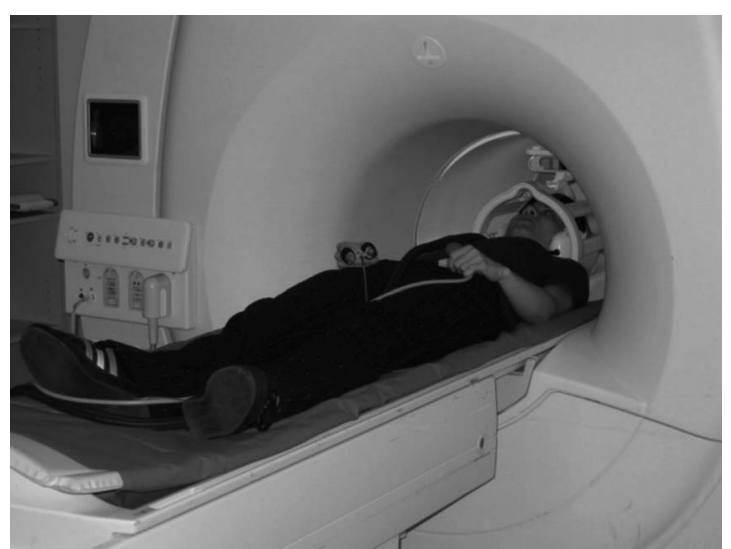

Fig. 3 Sensor positioning in an MR scanner during a neuroscience study

the sensor head. The measured light intensity from the sensor then changes linearly with the distance between the moving mirror and the sensor head. The advantage of the inclined double parallel structure is the translation from the grasping motion into vertical mirror motion toward the sensor head. By introducing this motion conversion, the optical fiber can be placed along the axis of the sensor and guided out along the scanner bed. Since the space is limited in an MRI scanner, this mechanical configuration significantly improves the accessibility of the sensor to the subject or patient, who is lying inside the scanner (Fig.3). This configuration also gives the sensor flexibility for its use in the MRI scanner. In addition, the sensor noise caused by extending, compressing, or bending of the fiber optic cable, can be minimized since the optical fiber is connected directly to the sensor in this configuration. Also, this configuration helps to minimize the sensor's size. As described in Section 2, the sensor structure is based on a compliant mechanism. Therefore, the sensor has a simple monolithic structure and is easily fabricated using a wire electrical discharge machine.

\subsection{Material}

To avoid electromagnetic interference, the sensor material was chosen carefully. In related research, some sensors have been made of plastic, brass, and aluminum. In this study, we made the sensor out of aluminum (A5052) in minimizing the sensor's volume in order to avoid eddy currents.

\section{Analysis}

Based on the structure described in Section 3, we analyzed the sensor structure using the Finite Element Method (FEM) to optimize the sensor design. The 3D model was meshed with tetrahedron elements using Femap 10.0.2[15] with a fundamental element size of $1.2 \mathrm{~mm}$. The FEM analysis was conducted using the DAFUL 3.3 mechanism analysis software[16]. 


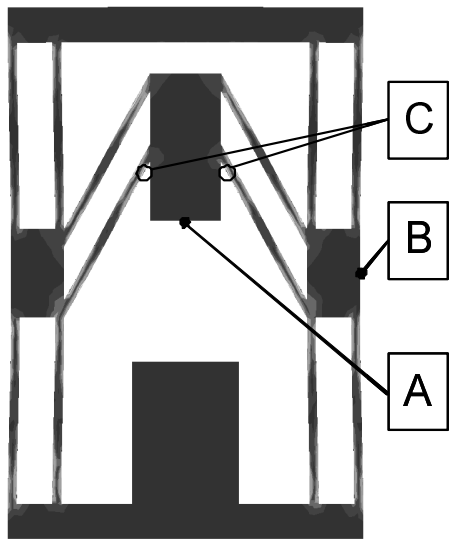

Fig. 4 Stress distribution resulting from FEM analysis

\subsection{Structural Optimization}

From the application requirements as a grasping sensor for human motor control investigations with fMRI, we determined the size of the sensor to be 30 $\times 20 \mathrm{~mm}$ with a thickness of $5 \mathrm{~mm}$. The required range of grasping force was up to $50 \mathrm{~N}$, and the required range of displacement was $0.1 \mathrm{~mm}$ (based on the fiber optic sensor). Based on these conditions, multiple iterations of FEM analyses were conducted, changing the thickness of the flexible beams that form the parallelograms. Finally, the thickness of the flexible beams was determined to be $0.5 \mathrm{~mm}$. Thus, the design parameters were determined quantitatively.

A representative FEM trial result for the stress distribution is shown in Fig. 4. From this result, it is shown that the stress is concentrated in the flexible beams of the parallelograms. Fig. 5 shows the analytical results for the mirror displacement (at position A and toward the sensor head) as a function of the applied grasping force. The results clearly show that the sensor has the desired linear relationship between the grasping force and the mirror displacement. From these results, the maximum displacement of the mirror toward the sensor head was determined to be approximately $0.1 \mathrm{~mm}$ (for $50 \mathrm{~N}$ of grasping force).

\subsection{Stiffness}

The stiffness of the sensor was investigated using FEM analysis. Fig. 6 shows an increase in the displacement of the grasping part (at position B) with an increase in the applied grasping force. From these results, the displacement at the maximum grasping force of $50 \mathrm{~N}$ was determined to be $0.17 \mathrm{~mm}$, and thus, the stiffness of the sensor was determined to be $294.1 \mathrm{~N} / \mathrm{mm}$. Therefore, the results reveal that the sensor is sufficiently stiff to be used in human motor control investigations.

\subsection{Maximum Applied Stress}

To test the sensor structure's robustness, we investigated the maximum stress that can be applied to the sensor. According to the series of FEM analyses, the maximum stress was at position C. The rela-

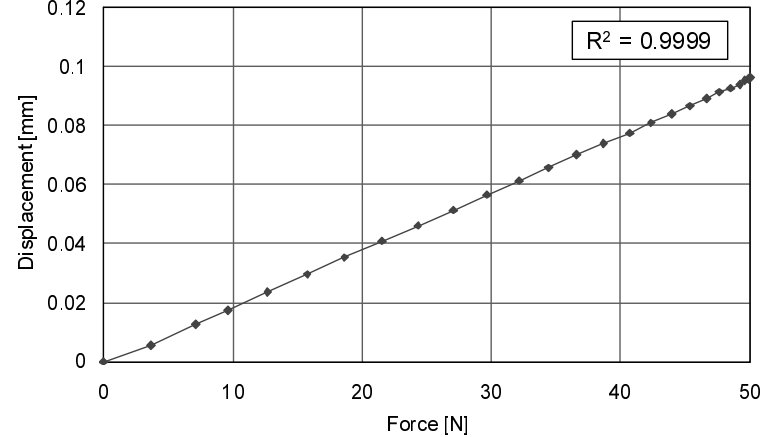

Fig. 5 Displacement at position A from FEM analysis

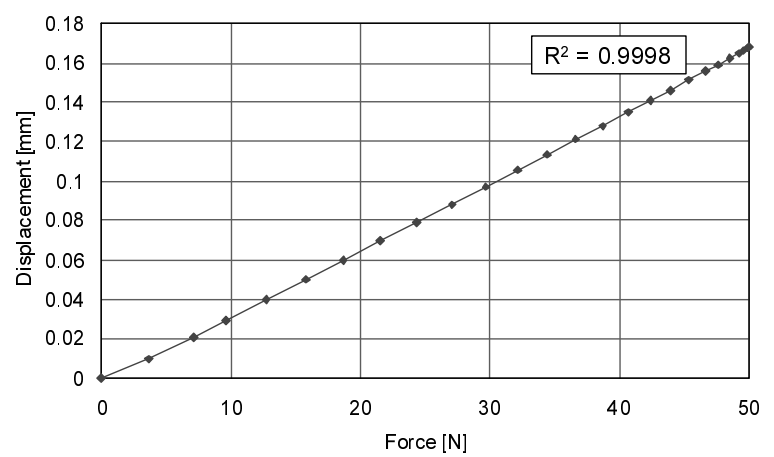

Fig. 6 Displacement at position B from FEM analysis

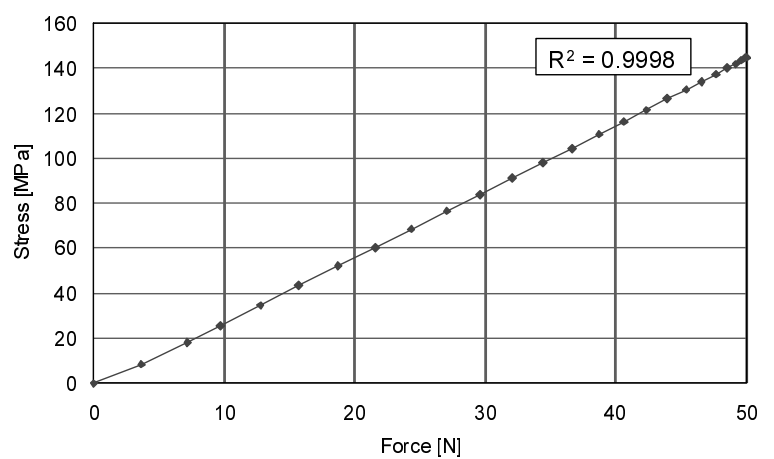

Fig. 7 Stress at position C from FEM analysis

tionship between the load and the stress at position $\mathrm{C}$ is illustrated in Fig. 7. The observed maximum stress at position $\mathrm{C}$ was $145 \mathrm{MPa}$ for an applied load of $50 \mathrm{~N}$. Since the $0.2 \%$ proof stress of A5052 is approximately $250 \mathrm{MPa}$, it is revealed that the sensor structure is still in elastic deformation and has no risk of fatigue failure.

\section{Characterization}

To evaluate the developed sensor, a calibration test and a grasping test were conducted using a prototype, and the results are discussed in this section. The evaluation tests were conducted in a non-MR environment. 


\subsection{Calibration Test}

A calibration test was conducted by applying a load to the grasping part of the sensor from both sides using a vice. The applied force was measured using a load cell fixed to the prototype sensor, as illustrated in Fig. 8. The load was increased from 0 to $20 \mathrm{~N}$, and the output voltage from the fiber optic sensor was recorded every $5 \mathrm{~N}$. The output voltage was measured by an AD conversion unit (AIO-163202SXUSB, Contec Co., Ltd., Japan[17]). The sensor output was recorded at $100 \mathrm{~Hz}$, and the average voltage of 100 data samples for each measurement is illustrated in Fig. 9. The bias voltage (approximately 4.1 V) was observed due to the initial distance between the mirror and fiber optic sensor, however, it does not effect on the measurement since we only focus on the voltage variation. From these experimental results, the following sensor characteristics are revealed.

Linearity: From the results, it is clearly shown that the developed sensor prototype has high linearity $\left(R^{2}=0.9996\right)$ due to the motion constraints of the newly developed parallel structure.

Resolution: From the results, the sensitivity of developed sensor was determined to be $0.038 \mathrm{~V} / \mathrm{N}$. It should be noted that, in this experimental range, the $\mathrm{AD}$ converter has a 16-bit resolution. Thus, the resolution of the prototype sensor was $0.002 \mathrm{~N}$, which is sufficiently high enough to conduct human motor control investigations.

Hysteresis: It is well known that sensors made of viscoelastic materials such as plastic typically have hysteresis. However, since the prototype sensor was made of aluminum, there was no hysteresis observed during the experiments.

From these results, it is shown that the developed sensor has suitable characteristics for use in human control investigations in an MR environment. Finally, the specifications of the developed MRI-compatible sensor are shown in Table 2.

\subsection{Grasping Test}

To test the prototype in a practical manner, a grasping test was conducted. In this test, the sensor was grasped by a hand, and the grasping force was exerted approximately three times every $2 \mathrm{~s}$ (Fig. 10). The experimental results are shown in Fig. 11 as the sensor output. The results clearly show that the sensor is working properly in repeated grasping trials. Therefore, the results show that the prototype can be used practically in human motor control investigations.

\section{Conclusions}

In this paper, we proposed a novel design of a grasping sensor to be used for precision grip tasks during fMRI. An MRI-compatible grasping sensor is a challenge in terms of safety, electromagnetic compatibility, size and accessibility (due to the limited MRI scanner space). The proposed sensor is equipped

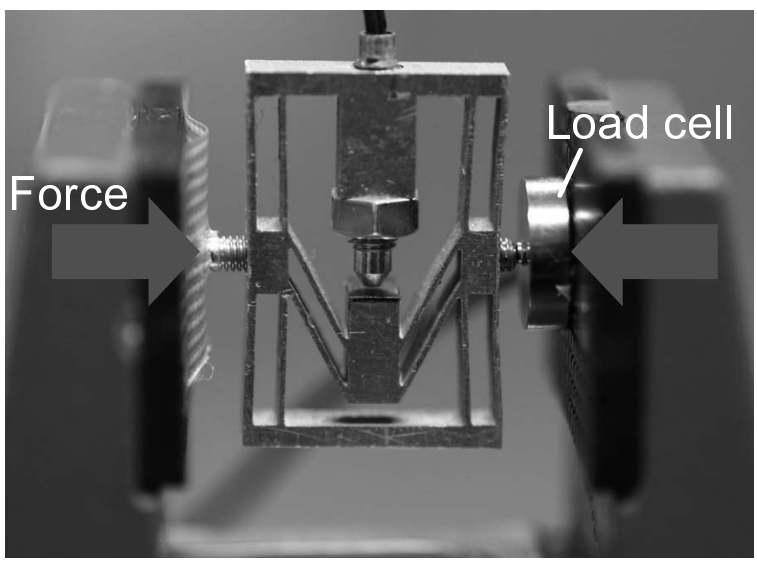

Fig. 8 Calibration test bench

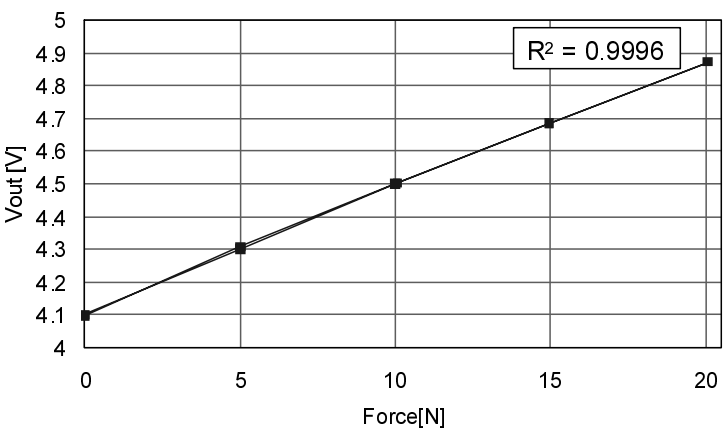

Fig. 9 Results of calibration test on the prototype

Table 2 Specifications of the developed MRI-compatible sensor

\begin{tabular}{l|l}
\hline Measurable force range & $0-50 \mathrm{~N}$ \\
\hline Resolution & $0.002 \mathrm{~N}$ \\
\hline Stiffness & $294.1 \mathrm{~N} / \mathrm{mm}$ \\
\hline Size & $30(\mathrm{H}) \times 20(\mathrm{~W}) \times 5(\mathrm{D}) \mathrm{mm}$ \\
\hline $\begin{array}{l}\text { Weight (including sen- } \\
\text { sor head) }\end{array}$ & $4.6 \mathrm{~g}$ \\
\hline
\end{tabular}

with a fiber optic sensor, which can measure the displacement in an MR environment. Using a newly developed inclined double parallel structure, the mirror displaces vertically (toward the sensor head) based on the grasping force. The developed inclined double parallel structure is composed of a pair of vertical parallelograms and a pair of inclined parallelograms. The main contribution of the paper is the novel sensor structure. Using this mechanism, the structure can translate the grasping motion into vertical motion of the mirror toward the sensor head. By introducing this motion conversion, the optical fiber can be placed along the sensor structure. Since the space is limited in an MRI scanner, this mechanical configuration improves the accessibility of the sensor to the subject or patient, who is lying inside the scanner. This configuration also gives the sensor flexibility for its use in the scanner. In addition, the sensor noise caused 


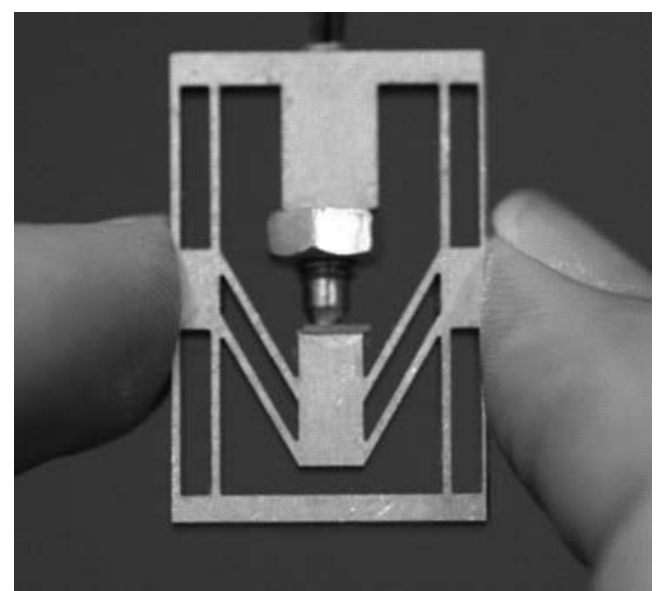

Fig. 10 Grasping test

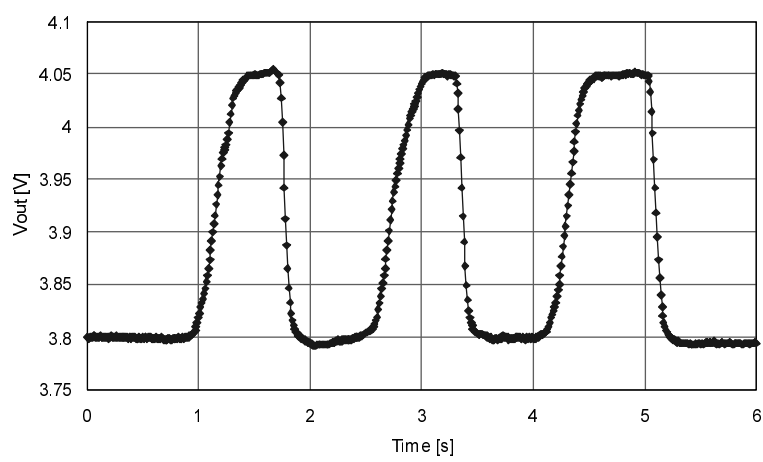

Fig. 11 Results of grasping test on the prototype

by extending, compressing, or bending the fiber optic cable can be minimized since the optical fiber is connected directly to the sensor in this configuration. Also, this configuration helps to minimize the sensor's size. Using the FEM analysis, the sensor's shape and dimensions were optimized. To evaluate the developed sensor, a calibration test and a grasping test were conducted using a prototype. Promising sensor characteristics were observed in these evaluation tests, including high linearity and negligible hysteresis.

\section{References}

[1] R. Gassert, D. Chapuis, H. Bleuler and E. Burdet: Sensors for applications in magnetic resonance environments; Proc. of IEEE/ASME Tran. on Mechatronics, Vol. 13, No. 3, pp. 335-344 (2008)

[2] R. Gassert, E. Burdet and K. Chinzei: MRcompatible robotics-A critical tool for image guided interventions, clinical diagnostics and neuroscience; Eng. Med. Biol. Mag., Vol. 8, No. 3, pp. 12-14 (2008)

[3] R. Gassert, E. Burdet and K. Chinzei: Opportunities and challenges in MR-compatible robotics; Eng. Med. Biol. Mag., Vol. 8, No. 3, pp. 15-22 (2008)

[4] U. X. Tan, B. Yang, R. Gullapalli and J. P. Desai: Design and development of a 3 -axis MRI-compatible force sensor; Proc. of IEEE Int. Conf. on Robotics and Automation, pp. 2586-2591 (2010)

[5] P. Puangmali, P. Dasgupta, L. D. Seneviratne and K. Althoefer: Miniaturized triaxial optical fiber force sensor for MRI-guided minimally invasive surgery; Proc. of IEEE Int. Conf. on Robotica and Automation, pp. 2592-2597 (2010)

[6] N. Takahashi, M Tada, J. Ueda, Y. Matsumoto and T. Ogasawara: An optical 6-axis force sensor for brain function analysis using fMRI; Tran. of the Japan Society of Mechanical Engineers, Series C, 70(691), pp. 743-750 (2004)

[7] M. Turkseven and J. Ueda: Design of an MRI compatible haptic interface; Proc. of IEEE/RSJ Int. Conf. on Robotics and Systems, pp. 2139-2144 (2011)

[8] T. Tokuno, M. Tada and K. Umeda: High-precision MRI-compatible force sensor with parallel plate structure; Proc. of IEEE/RAS-EMBS Int. Conf. on Biomedical Robotics and Biomechatronics, pp. 33-38 (2008)

[9] P. Polygerinos, P. Puangmali, T. Schaeffter, R. Razavi, L. D. Seneviratne and K. Althoefer: Novel miniature MRI-compatible fiber-optic force sensor for cardiac catheterization procedures; Proc. of IEEE Int. Conf. on Robotics and Automation, pp. 25982603 (2010)

[10] Y. L. Park, K. Chau, R. J. Black and M. R. Cutkosky: Force sensing robot fingers using embedded fiber bragg grating sensors and shape deposition manufacturing; Proc. of IEEE Int. Conf. on Robotics and Automation, pp. 1510-1516 (2007)

[11] L. L. Howell: Compliant Mechanisms, WileyInterscience (2001)

[12] N. Lobontiu: Compliant Mechanisms, CRC Press (2002)

[13] S. T. Smith: Elements of Elastic Mechanisms, CRC Press (2000)

[14] J. P. Merlet: Parallel Robots Second Edition, Springer, ISBN 1-4020-4132-2 (2006)

[15] SIEMENS, Femap, http://www.plm.automation. siemens.com/en_us/products/velocity/femap (Nov. 2011)

[16] Virtual Motion, DAFUL, http://www. virtualmotion.co.kr/eng/product_01.html (Nov. 2011)

[17] AD conversion unit AIO-163202SX-USB http:// www.contec.com/product.php?id=2056 (Nov. 2011)

Jumpei ARATA

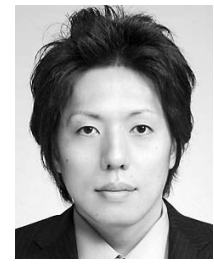

\section{Authors}

received the $\mathrm{Ph}$. D. degree in mechanical engineering from the University of Tokyo, Tokyo, Japan in 2004. Since 2004, he has been an Assistant Professor at the Nagoya Institute of Technology, Nagoya, Japan. His research interests are parallel mechanism and compliant mechanism, and these applications in industrial robot, haptic device, surgical robot and rehabilitation robot. 


\section{Shogo TERAKAWA}

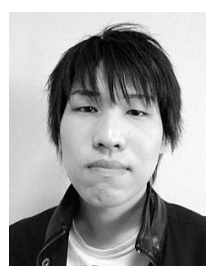

received the Undergraduate Degree in Mechanical Engineering and the Master Degree in Mechanical Engineering from Nagoya Institute of Technology, Nagoya, Japan in 2010 and 2012 respectively. His research interests are mechanical design and implementation of medical sensor.

\section{James SulzER}

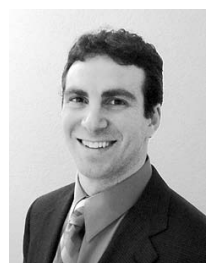

received his Ph.D. in Mechanical Engineering from Northwestern University and the Rehabilitation Institute of Chicago, Chicago, United States, in 2009. He is currently a postdoctoral fellow in the Rehabilitation Engineering Lab at the ETH Zurich, Zurich, Switzerland. He is interested in sensorimotor control and learning, technologyaided rehabilitation, medical robotics and neuroimaging.

\section{Roger GASSERT}

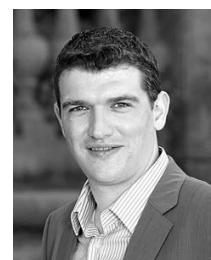

received the M.Sc. degree in microengineering and the Ph.D. degree in neuroscience robotics from the Ecole Polytechnique Fédérale de Lausanne (EPFL), Lausanne, Switzerland, in 2002 and 2006, respectively. Since 2008, he has been an Assistant Professor of rehabilitation engineering with ETH Zurich, Zurich, Switzerland. His research interests are in physical human-machine interaction, rehabilitation robotics, assistive technology, and the neural control of movement.

\section{Hideo Fujimoto}

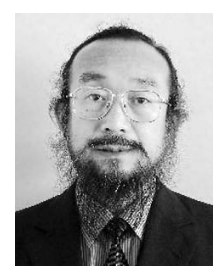

received an undergraduate degree and Ph.D. degree in mechanical engineering from Nagoya University, Nagoya, Japan in 1970 and 1982, respectively. $\mathrm{He}$ is currently a professor at the Nagoya Institute of Technology, Nagoya, Japan. His research interests are medical engineering, haptic engineering, and robotics. 